\title{
A virtual reality exposure therapy for ptsd patients controlled by a fuzzy logic system
}

\author{
Fernando M. de Oliveira (UERJ) \\ Regina S. Lanzillotti (UFRJ) \\ Rosa. M. E. M. da Costa (UERJ) \\ Raquel Gonçalves (UFRJ) \\ Paula Ventura (UFRJ) \\ Luís. A. V. de Carvalho (UFRJ)
}

\begin{abstract}
This paper describes the main characteristics of two integrated systems that explore Virtual Reality technology and Fuzzy Logic to support and to control the assessment of people with Post-Traumatic Stress Disorder during the Virtual Reality Exposure Therapy. The integration of different technologies, the development methodology and the test procedures are described throughout the paper.
\end{abstract}

Keywords: Virtual Reality, Post-Traumatic Stress Disorder, Exposure Therapy, Fuzzy Logic.

\section{Introduction}

Recently, the increasing production exploring the Virtual Reality technologies is undeniable, particularly in the area of Medical Science. Although the three-dimensional (3-D) virtual environments have been frequently used in the neuropsychological area, the use of intelligent strategies for monitoring the patients' activities is rare. In general, the three-dimensional (3-D) environments open new possibilities to create environments suitable for simulations in the rehabilitation processes of cognitive functions loss caused by traumas and injuries. These environments allow the simulation of real situations integrated with psychological evaluations, proposing tasks "with low risks to the patient". These characteristics stimulate the growing interest in the Virtual Reality Exposure Therapy (VRET) applications.

Many people suffer different traumatic events, but only $10 \%$ to $60 \%$ of men and $50 \%$ of women develop lifelong Posttraumatic Stress Disorder (PTSD) (Masci and Range, 2001). PTSD involves a constant sense of fear generated by the improper consolidation of trauma in the autobiographical memory (Brewin, Dalgleish and Joseph, 1996). Foa and Kozak (1986) argued that some exposure strategies to feared situations are common in psychotherapies for anxiety and that confrontation is an effective treatment for the anxiety disorder. However, for some patients it is difficult to immerse themselves in a scene due to avoidance traumatic symptoms. Thus, the use of new technologies could facilitate the treatment of these patients. In this sense, virtual reality has been used as a tool for exposure and it has achieved positive results in the treatment of several anxiety disorders, including Specific Phobias, Social Phobia, Panic Disorder and PTSD (Meyerbröker and Emmelkamp, 2010).

This paper presents two integrated environments for PTSD treatment: the ARVET (Virtual Environment Exposure to Trauma), a 3-D virtual environment to stimulate the memory of the patient with PTSD and the SAPTEPT (Evaluation System to Patients with Posttraumatic Stress Disorder), which explores the Fuzzy Logic to support the patients evaluation submitted to the ARVET. The ARVET presents some scenes that incorporate stimulus to the emotional processing, allowing an increase or decrease of the emotional answer. The SAPTEPT combines the perceived anxiety, indicated by the patient and the cardiac rate to classify the patient's level of anxiety as mild, moderate or severe. This classification will help the therapist to control the stimulus level provided by the ARVET environment. 
This work is organized into 4 sections. Section 2 describes the main concepts related to the proposed systems; section 3 details the environment and presents the expected interaction results between the systems. Section 4 concludes the work and presents future research directions.

\section{General Concepts}

\section{PTSD Treatment}

PSTD involves a constant sense of fear generated by the improper consolidation of autobiographical memory of the trauma (Brewin, Dalgleish \& Joseph, 1996). People with PTSD can not adapt back to their usual life. Thus, a re-reading of the environment they live in will be fundamental to reorganize the experienced situations.

In recent years, different theoretical and practical approaches have been explored to assess and rehabilitate cognitive processes such as visual perception, attention and memory, as well as training the motor skills (Ready, Pollack \& Rothbaum, 2006). The Cognitive-Behavioral Therapy (CBT) is considered a good option for Post-traumatic Stress Disorder (PTSD) treatment. In general, it is performed by exploring techniques that include Psycho Education, Cognitive Restructuring Techniques, Anxiety Management, Imaginary Exposure and Live Exposure. Its aim is to break the cycle of symptoms leading to a habituation of stimuli. Moreover, it attempts to develop relaxation skills, enabling the patients' control of their emotional and physiological response in order to decrease their reaction when faced with stressful situations.

\section{Virtual Reality}

The Virtual Reality technology has been widely used in the cognitive stimulation, providing opportunities to offer some situations closer to the real world. Burdea and Coiffet (2003) defines the applications of Virtual Reality as a three-dimensional virtual environments that presents real-time graphics rendered by a computer, in which the user, via body position sensors or user-input devices, controls the viewpoint or the orientation of displayed objects.

In the last years virtual reality exposure therapy (VRET) has become a viable alternative for exposure in vivo, the gold standard for the treatment of anxiety disorders (Meyerbröker and Emmelkamp, 2010). In spite of exposure therapy, some patients find it difficult to imagine themselves in the scene due to avoidance symptoms, leading some of them to abandon treatment. In some studies, dropouts and non-response rates can reach $50 \%$ of cases (Schottenbauer, Glass, Arnkoff \& Gray, 2008). Therefore, the use of new technologies could smooth the progress of exposure to avoidant patients. In this sense, virtual reality has been used as a tool for exposure and it has achieved positive results for treatment of several anxiety disorders, including Specific Phobias, Social Phobia, Panic Disorder and PTSD (Meyerbröker and Emmelkamp, 2010).

The Virtual Reality Exposure Therapy (VRET) facilitates the emotional engagement of patients with PTSD during exhibitions, outlining the avoidance symptoms, and therefore facilitates the therapist control (Ready, 2006). According to Rothbaum and Mellman (2001), the sense of presence induced by the virtual environment, which is rich in sensory stimuli, helps processing the emotional memories related to trauma. This technological device allows gradual exposure to the feared environment, according to patient needs. In this sense, some experiments have been done worldwide. Torres and Nunes (2011) used three-dimensional virtual environments with characteristics of "Serious Games" to simulate situations aimed at training and therapy. These games allow the simulation of real-world situations, providing training activities that stimulate cognitive functions and psychomotor skills. The study conducted by McLay, Wood and Webb-Murphy (2011) evaluates the effectiveness of the VRET in the treatment of PTSD patients on the spot to combat in Iraq. According to these authors, three-dimensional virtual environments can be used to simulate situations aimed at training and therapy. In general, there is no automated control that gives the therapist results from the integration of different biofeedback variables obtained during the VRET. As an alternative, the inclusion of intelligent techniques, as Fuzzy Logic, can help to alleviate 
this problem by reducing the need of therapist decisions.

\section{Fuzzy Logic}

According to Zadeh (Zadeh, 2009) Fuzzy logic adds to bivalent logic an important capability: to reason precisely using imperfect information. Imperfect information is information which in one or more dimensions is imprecise, uncertain, incomplete, unreliable, vague or partially true. Fuzzy Logic uses the formal principles of approximate reasoning and searching to model imprecise reasoning that are usual in the human decisions. It has been a powerful tool, which is able to capture inaccurate information described in natural language and translate them to a qualitative and quantitative form, allowing the position of super categories.

Traditionally, a logical position has two extremes: "true" or "false". However, in fuzzy logic, a premise varies in degree of truth or relevance in the range $0-1$, which leads to concepts of partly true or partly false. The control performed by the fuzzy logic mimics behavior-based rules, rather than a control explicitly restricted to deterministic models. The goal of Fuzzy Logic is to generate a logic output from a set of non-precise inputs, noisy, incomplete or even absent ones. According to Braga, Barreto and Machado (1995), "Fuzzy Math is an attempt to bring together the characteristic precision of mathematics and the inherent imprecision of the real world, born from the deep desire to better understand the mental processes of reasoning".

Fuzzy Logic has been successfully exploited as a tool to support the analysis of tests of three-dimensional environments (3-D), devoted to the teaching and training of medical practices (Santos, Machado, Moraes \& Gomes, 2011).

\section{The ARVET and SAPTEPT Systems}

Usually, the treatment of a patient with PTSD, provided by the Stress Research Laboratory (Laboratorio de Pesquisa Integrada do Estresse LINPES-UFRJ) explores two stimulation strategies: imaginary and live. In imaginary exposure, the patient informs the details of the trauma in a sequential manner at each visit. In this case, the patient is exposed to the reported trauma listening to an audio recorder. In the live exposure the intention is a direct and graduated confrontation to the feared objects or situations.

To support the LINPES practices we developed two applications to help both therapists and patients in the live exposure process. First, we constructed a hierarchy of feared situations to implement a collection of virtual environments to support the exposure to different levels of intensity: the ARVET - Environment Virtual Reality Exposure to Trauma. The ARVET is a collection of virtual reality environments that were built with the possibility of stereoscopic viewing through a large screen and the use of 3-D glasses, which interact with the patient and stimulate them. The tools used to create this environment were: Blender 2.5 (www.blender.org) to model objects in the scene and Unity3D 3.4.2 (unity3d.com) for the animations and interactions. The first prototype simulates some situations involved in urban violence. Figures 1 and 2 show some images of ARVET. 


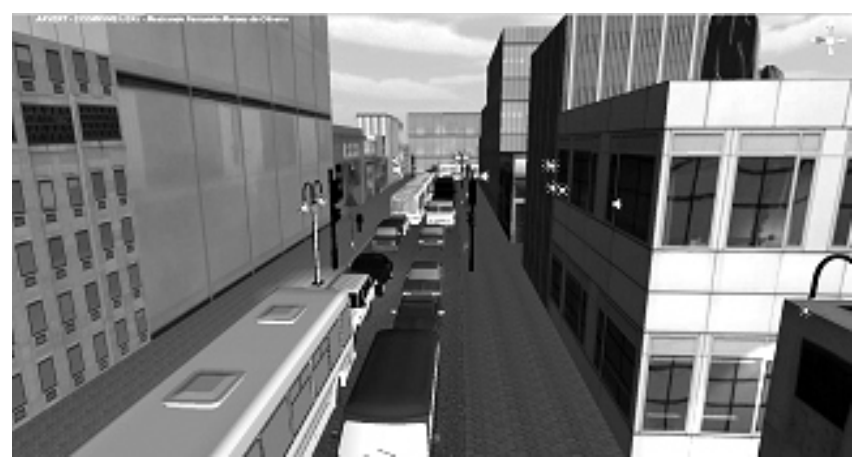

Figure 1: The ARVET environment: general views

The exposure to virtual environments can generate reactions and perceptions, which are difficult to be assessed jointly by the therapist. Aiming to integrate these data and classify the patient in real time during the VRET process, we developed an application that explores the techniques of Fuzzy Logic - the SAPTEPT System of the Evaluation of the Patients with Posttraumatic Stress Disorder.

The LINPES researchers distinguish the groups of Psychometric and Psycho-physiological variables, whose values are collected during evaluations. Psychometric scales are established by filling in forms of self-report and Psycho-physiological data are obtained through the Biopac (Physiological data acquisition system).

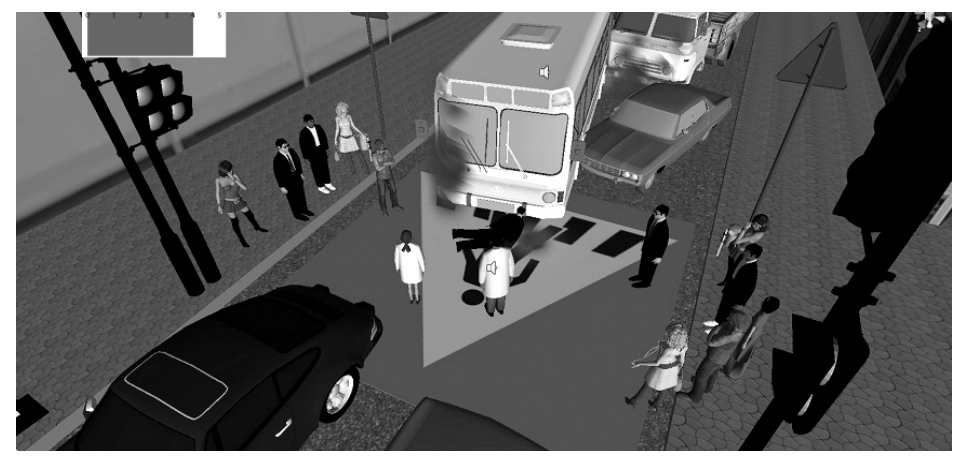

Figure 2: The scene of an accident: a person was hit by a bus

The scale used to measure the patient level of anxiety is the SUDS - Subjective Units of Disturbance Scale that assesses the degree of anxiety during the trauma stimulation. The SUDS is a range of integer values between 0 and 10 that measures the intensity of disturbance or distress experienced by an individual when subjected to trauma. For example, the individual perceives their level of anxiety at the time of exposure to the trauma, ranging from "no anxiety" to "high anxiety".

A meta-analysis conducted by Pole (2007) identified the Heart Rate as a Psycho-physiological group variable that changes when PTSD patients are exposed to a traumatic stimulus. Thus, the heart rate level confirms or not, the patient's anxiety. In this case, we consider the level of anxiety reported by the patient and the heart rate. Table 1 presents these variables. 


\begin{tabular}{|l|l|}
\hline Psychometric Scales & Psycho-physiological Variables \\
\hline Anxiety & Heart Rate \\
\hline
\end{tabular}

Table 1. Variables involved in the SAPTEPT system.

The Fuzzy Logic used in SAPTEPT captures inaccurate information, described them in natural language and converts them in qualitative information. The Fuzzy Logic can control the behavior of a system by changing the inputs according to a set of inference rules. These behavior-based rules model the system operations. The method, from the viewpoint of Fuzzy Logic, allows us to recognize patterns of anxiety gradual scale (Mild, Moderate, and Severe) in real-time measurements, when the psychometric scales (anxiety) and psycho-physiological (heart rate) are performed (Figure 3). Pattern recognition is one of the oldest and most obvious applications in the area of Fuzzy Theory.

This system classifies the patient's degree of anxiety while they are navigating in the ARVET. It indicates the difficult level changes that must be performed in the ARVET scenario. All tasks, scenes and the patient classification in the Fuzzy System were proposed by a group of psychologists, which are responsible to test these systems with their patients.

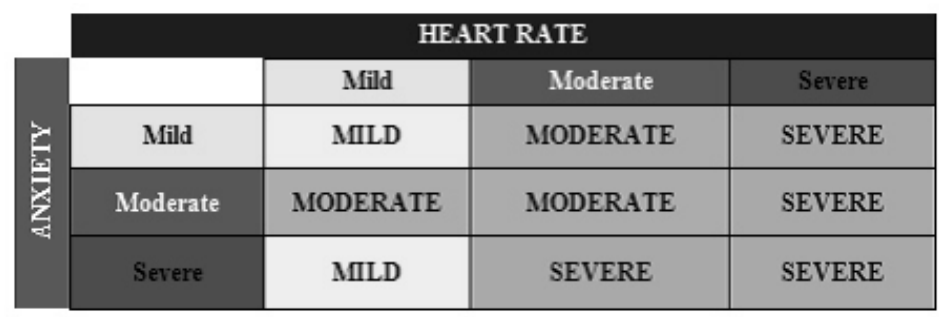

Figure 3. Fuzzy Inference table

The SAPTEPT was developed in Python programming language and shows the classification of the patient every five minutes from the start of the evaluation. The results form the SAPTEPT system must be monitored by a therapist.

After the patient is classified in a new level, the therapist will ask him to open a door and enter in a new scene (Figure 4) that will present situations according to the patient classification.

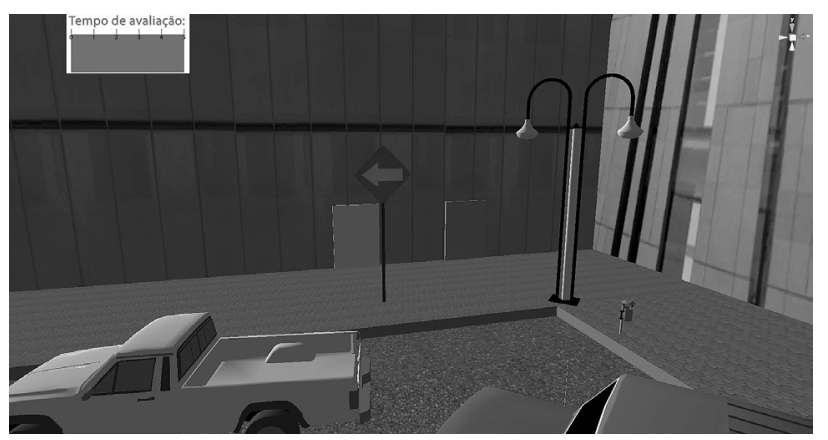

Figure 4. The doors where the patient must enter to change the level of stimulation 
A pipeline of the system use is presented in Figure 5.

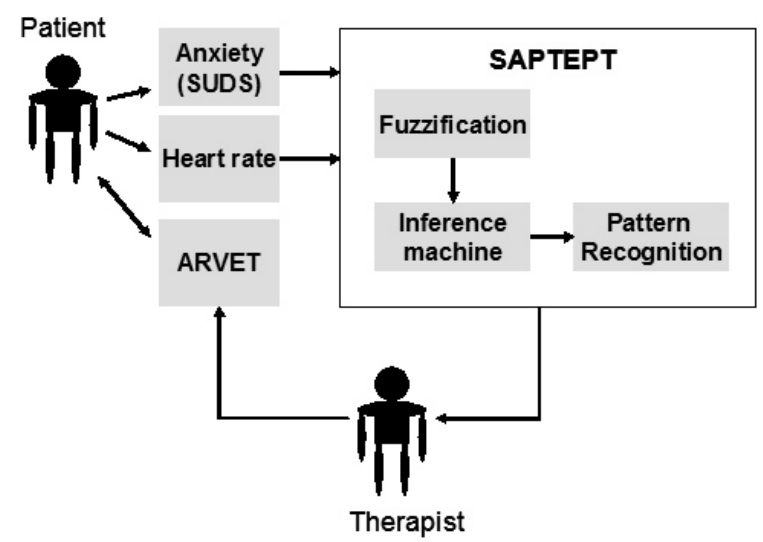

Figure 5. The pipeline of the system use.

\section{Perspectives}

Aiming at analyzing some aspects of the prototype, we developed an evaluation experiment with two psychologists. This evaluation considered some usability aspects as: navigation facility; learning facility; response time; realism of scenes; pleasantness of the scenes; adequacy of objects in the tasks and matching colours.

The initial results of this experiment indicated that the 3-D environment has a high level of usability, but some aspects must be changed: some colors, some architectural details and the speed of buses and cars. At the other side, the Fuzzy system must have its interface slightly modified in order to facilitate responses from users about their anxiety level.

After those steps, the system will be used by a group of people with Posttraumatic Stress Disorder to measure its efficacy in control the level of tasks according to the patient evolution.

The prototype can be used in the first instance for cases of patients diagnosed with PTSD due to hits or car accidents (very common in large urban centers).

\section{Conclusions}

In Brazil, there is a growing interest in the Virtual Reality technology to support health care procedures. In this sense, we need new software and new treatment strategies, where patients may have unrestricted access to the exercises with therapists precisely monitoring the results. Thus, in this case, the virtual environment must have some mechanisms to control user navigation and generate automatic reports to the therapist.

However, the development of such software depends on the integration of different technologies and expertise. In this context the Fuzzy Logic offers wide possibilities to control the user answers and support the therapist decisions.

This paper presented some results of a project that has two objectives. The first one is associated with the technical questions related to the intelligent strategies to support decisions and the second deals the integration of the intelligent modules with specific virtual three-dimensional environments. The next step of this research is to integrate the SAPTEPT with the ARVET.

Some therapists (psychologists) tested the system and proposed some changes that were considered in a new 
version of the system. The system is currently being tested with a group of people with PSTD associated to car accidents.

\section{Acknowledgements}

This study is supported in part by the FAPERJ (Fundação Carlos Chagas Filho de Amparo à Pesquisa do Rio de Janeiro), Brazil.

\section{References}

[1] Burdea, G., Coiffet, P. (2003), Virtual Reality Technology. New Jersey: John Wiley \& Sons, 2a Ed.

[2] Braga, M., Barreto, J. M. Machado, M. A. (1995), Conceitos da Matemática Nebulosa na Análise de Risco. Ed. Artes\& Rabiskus, (in Portuguese).

[3] Brewin, C. R., Dalgleish, T., Joseph, S. A. (1996), Dual representation theory of post traumatic stress disorder. Psychological Review, 103, 670-686.

[4] Foa, E. B., Kozak, M. J. (1986), Emotional Processing of Fear: Exposure to Corrective Information, Psychological Bulletin, 99(1), 20-35.

[5] Masci, C., Range, B. (2001), Transtorno de Estresse Pós-Traumático, Psicoterapias Cognitivo-Comportamentais. Range B. (Org.), ArtMed Editora, (in Portuguese).

[6] McLay, R. N., Wood, D. P., Webb-Murphy, J. A. (2011), A Randomized, Controlled Trial of Virtual Reality-Graded Exposure Therapy for Post-Traumatic Stress Disorder in Active Duty Service Members with Combat-Related Post-Traumatic Stress Disorder. Cyberpsychology, Behavior, and Social Network, 14(4), 223-229.

[7] Meyerbröker, K., Emmelkamp, P. M. (2010), Virtual reality exposure therapy in anxiety disorders: a systematic review of process -and-outcome studies. Depression and Anxiety, 27, 933-944.

[8] Pole, N. (2007), The Psychophysiology of Posttraumatic Stress Disorder: A Meta-Analysis. Psychological Bulletin, 133(5), 725746.

[9] Ready, D. J., Pollack, S., Rothbaum, B.O. (2006), Virtual reality exposure for veterans with posttraumatic stress disorder, J Aggression, Maltreatment, \& Trauma, 12, 199-220

[10] B. Rothbaum, O. (2001), Dreams and exposure therapy in PTSD, Journal of Traumatic Stress, 14, 481-490.

[11] Santos, A.D., Machado, L. S., Moraes. R. M., Gomes, R.G.S. (2011), Avaliação baseada em Lógica Fuzzy para um Framework voltado a construção de simuladores baseados em RV, XIII Simposium on Virtual and Augmented Reality, Natal, Brazil. 194-202 (in Portuguese).

[12] Schottenbauer, M.A., Glass, C.R., Arnkoff, D.B., Gray, S.H. (2008), Contributions of psychodynamic approaches to treatment of PTSD and trauma: a review of the empirical treatment and psychopathology literature. Psychiatry. 71(1), 13-34.

[13] Torres, R.S., Nunes, F. L. S. (2011), Aplicando aspectos lúdicos de Serious Game em treinamento médico: revisão sistemática e implementação, XIII Simposium on Virtual and Augmented Reality, Uberlândia, Brazil, 1, 1-8 (in Portuguese).

[14] Zadeh, L. (2009), Toward extended fuzzylogic- A first step, Fuzzy Sets and Systems, 160(21), 3175-3181. 